\title{
SRT1720 Protects Against CSE-Induced Cellular Senescence via Accelerates of F0X03-PINK1- mediated Mitophagy
}

hui Jiang

Zhejiang Provincial People's Hospital

Changyong Fu

Tongde Hospital Of Zhejiang Province

Dong Yuan

Zhejiang Provincial People's Hospital

Yaona Jiang

Zhejiang Provincial People's Hospital

Yaqing Li ( $\square$ lidoctor03@126.com )

Cancer Hospital of the University of Chinese Academy of Sciences https://orcid.org/0000-0002-73798151

Research

Keywords: Sirt1, SRT1720, selisistat, COPD, mitophagy, Foxo3A, PINK1

Posted Date: May 13th, 2021

DOI: https://doi.org/10.21203/rs.3.rs-482635/v1

License: (c) (1) This work is licensed under a Creative Commons Attribution 4.0 International License.

Read Full License 


\section{Abstract}

\section{Background}

Chronic obstructive pulmonary disease (COPD) is often associate with cigarette smoke extract (CSE)introduced bronchial epithelial cell senescence, mitochondrial fragmentation. Sirtuin-1(Sirt1) has been reported to play a crucial role in mitochondrial homeostasis and confers a protective role against the onset and development of CSE introduced bronchial epithelial cell senescence in COPD although the precise mechanism(s) remain elusive. Here we hypothesized that SRT1720, a pharmacological SIRT1720 activator, exerts protect against COPD by activating PINK1 mediated mitophagy, en route to preserved mitochondrial homeostasis.

Methods

COPD rats model was established by CS exposure. During 6 months of SRT1720 treatment, airway resistance, cellular senescence and mitochondrial injury, mitophagy in the lung tissues of model rats were examined by western blot(WB) and histochemical and immunofluorescence staining. Transmission electron microscopy was also carried to elucidate the effects of SRT1720.Human bronchial epithelial cells(HBEC) were used to clarify the underlying molecular mechanisms.

Result

During the introduction of CSE in cellular or rats, administration of SRT1720 improved airway resistance, cellular senescence and mitochondrial injury, accompanied with suppressed autophagy and mitophagy. Mitochondrial damage, cellular senescence and lung injury under contrast exposure were more severe in FOXO3 or Pink1 deficient cells and mice than in SRT1720 groups. Activation of Sirt1 by treating with SRT1720 induces autophagy enhanced. A Decrease in sirt1 expression caused by selisistat treatment promotes senescence.

\section{Conclusions}

Taken together, our data suggested that suppressed SIRT1/FOX03/Pink1 signaling mediated mitophagy played a protective role in COPD by reducing mitochondrial reactive oxygen species (ROS).

\section{Background}

Chronic obstructive pulmonary disease (COPD) is one of the most common chronic and disabling diseases and a growing cause of morbidity and mortality[1]. It is currently the fourth leading cause of death worldwide, and the World Health

Organization (WHO) predicts that it will become the third leading cause by 2030. In general, COPD is classified as chronic inflammatory disease associated cell metabolism disorder resulting from cigarette smoke, oxidative stress and inflammatory injury[2-4]. Accelerated cellular senescence resulting from 
cigarette smoke (CS) exposure with excessive reactive oxygen species (ROS) production has been implicated in the pathogenesis of COPD[5]. Nonetheless, the precise molecular mechanism(s) involved in the pathogenesis of COPD remains elusive.

Mitochondria, an organelle that maintains cell energy metabolism, is highly involved in cerebral ischemia/reperfusion injury[6]. The stability of its structure and function is considered an important therapeutic target. The process by which cells clear damaged or dysfunctional mitochondria and complete self-renewal to maintain mitochondrial quality control is called mitophagy[7, 8]. The autophagy and renewal of mitochondria are the prerequisites for repetitive operation and continuous supply of energy to cells[9]. Moderate mitophagy can protect mitochondrial function and help maintain cell energy metabolism and survival, excessive or insufficient autophagy will paralyze mitochondria and lead to cell death[10].

Sirtuin deacylase enzymes are important modulators of mitochondrial energy metabolism. Sirtuin 1(SIRT1) is the mammalian orthologue of the yeast silent information regulator 2 (Sir2), which is function as NAD+-dependent deacetylases as well as ADP ribosyltransferases[11-13]. SIRT1 can modulates the activity of deacetylates histone and transcription factors such as p53 and forkhead box 03 (Fox03)[14, 15].Emerging evidences have shown sirt1, an anti-inflammatory and anti-aging protein, is reduced in lungs of patients with COPD[16]. Recently evidence has depicted the molecular mechanism of CSinduced cellular senescence via a SIRT1-FOXO3 axis protects against stress-induced premature senescence (SIPS) and various pathophysiological changes in COPD.[17]

SRT1720, a pharmacological Sirt1 activator, was found a positive regulator for emphysema via upregulated the levels of foxo3, increased SIRT1 activity and inhibited AECII apoptosis[18-20]. Further studies also depicted a novel role for sirtuins in the pathogenesis and treatment of COPD. Moreover, the network of SIRT1-FOX03-Pink1 was reported to govern mitophagy and mitochondrial balance between fusion and fission[21]. However, the role of Sirt1 in the regulation in COPD has not been fully elucidated.

In this context, this work was designed to determined (a) whether SIRT1 imposes any effect on the regulatory role of autophagy in cigarette smoke extract (CSE)-induced cell senescence of primary HBEC, if any;(b) whether the foxo3a-pink1 signaling pathway is involved in mitophagy regulation triggered by SIRT1.

\section{Materials And Methods}

\section{Mice}

We purchased 45 male C57B1/ 6 wild type mice $(8$ weeks,200 $\pm 25 \mathrm{~g})$ from Shanghai Laboratory Animal Co., Ltd.(shanghai, China).We maintained the mouse at $21^{\circ} \mathrm{C}-25^{\circ} \mathrm{C}$ and $40 \%-60 \%$ relative humidity in a $12-$ hour light/dark circle and provided them with water and food ad libitum in the Laboratory Animal Center of Zhejiang Industry University(Hangzhou, China).The mice were randomly sorted into 4 groups(15

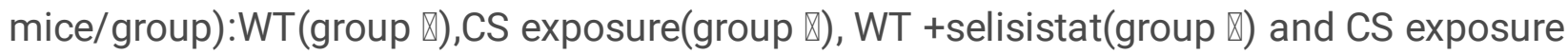


+selisistat(group 区).All animal protocols were approved by the Institutional Animal Care and Use Committee of Zhejiang Chinese Medical University.

\section{CS exposure}

A custom-designed cigarette smoking chamber stimuli were used for generating the mice model of COPD. For studies involving 3 days of CS exposure, research grade cigarettes (Xiongshi; China Tobacco Zhejiang Industrial. Co., Ltd., China) were used to generate smoke. Briefly, cigarette smoke exposure were

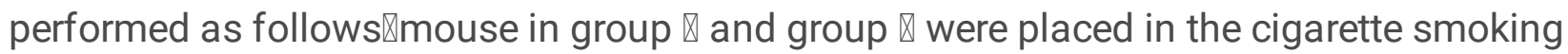
chamber $(60 \mathrm{~cm} \times 50 \mathrm{~cm} \times 40 \mathrm{~cm})$. After an interval of 10 minutes, the smoke of 5 new cigarettes was delivered into the chamber. CS concentration was set at a value of approximately $300 \mathrm{mg} / \mathrm{m}^{3}$ total particulate matter(TPM) by adjusting the flow rate of the diluted medical air, and the level of carbon monoxide in the chamber was $350 \mathrm{ppm}[22,23]$, monitored by a real time aerosol monitor(MicroDust pro,Casella CEL, Bedford, UK). The mouse were exposed to smoke for 90 minutes daily for consecutive 24 weeks.

\section{Administration of selisistat}

Selisistat(30mg/kg body weight in 10\%DMSO and 40\%PEG300 and 5\%Tween-80 and $45 \%$ saline,>95\% pure by C-13 NMR and LCMS; synthesized by life chemicals) through oral gavage 1 hour prior to CS exposure daily. To study the therapeutic effect on emphysema, selisistat was orally administrated daily for 4 weeks after the development of CS induced emphysema.

\section{Cell culture}

Normal airways were obtained from 4st order bronchi from pneumonectomy and lobectomy specimens for primary lung cancer. Informed consent was obtained from all surgical participants as part of an approved ongoing research protocol by the ethics committee of Hangzhou medical college. HBEC were isolated with protease treatment and characterized as previously describe[24]. HBEC were serially passaged and used for experiments were performed with HBEC from non-COPD patients. HBEC was cultured in RPMI1640 with 10\% fetal calf serum and penicillin-streptomycin(Gibo Life Technologies,15140-112).For in vitro study, HBEC were treated with CSE(1\%) and SRT1720(4mM/L,>95\% pure by C-13 NMR and LCMS; synthesized by life chemicals)for $48 \mathrm{~h}$.

\section{Preparation of cigarette smoke extract(CSE)}

Cigarette smoke extract (CSE) was prepared as previously described with minor modification[10]. Forty milliliters of cigarette smoke were drawn into the syringe and slowly bubbled into sterile serum-free cell culture media in 15-ml BD falcon tubes. One cigarette was used for the preparation of $10 \mathrm{ml}$ of solution. CSE solution was filtered(0.22 $\mu$ m;Merck Millipore,SLGS033SS) to remove insoluble particles and was designated as a $100 \%$ CSE solution.

\section{Measurement of lung mechanics}


Lung mechanical properties, including lung compliance and RL, were determined as described previously[25]. Briefly, the mouse was weighed, deeply anesthetized by i.p. injection of pentobarbital (90 $\mathrm{mg} / \mathrm{kg} \mathrm{BW}$ ) and pancuronium ( $0.5 \mathrm{mg} / \mathrm{kg} \mathrm{BW})$, and tracheostomized. The trachea was cannulated, and the cannula was connected to a computer-controlled small animal ventilator (FlexiVent; SCIREQ).

\section{Lung morphometry}

Mouse lungs (which had not been lavaged) were inflated with 1\% low-melt agarose at a pressure of 25 $\mathrm{cm} \mathrm{H} 20$, then fixed with $4 \%$ neutral buffered PFA $[25,26]$. Fixed lung was dehydrated, embedded in paraffin, and sectioned into $4-\mu \mathrm{m}$ sections using a rotary microtome (MICROM International GmbH). H\&E staining was performed on the lung midsagittal sections to determine Lm of airspace using MetaMorph software (Molecular Devices) [25]. Ten randomly selected $\times 100$ fields per slide were photographed in a blinded manner, and the images were manually thresholded. The airway and vascular structures were eliminated from the analysis.

\section{Senescence-associated- $\beta$-galactosidase (SA- $\beta$-gal) activity assay}

SA- $\beta$-gal activity was assessed using an in situ $\beta$-galactosidase staining kit (Beyotime Institute of Biotechnology, Shanghai, China) according to the manufacturer's protocol. Lung tissues were fixed in $\beta$ galactosidase stationary solution for $15 \mathrm{~min}$, then washed 3 times for $10 \mathrm{~min}$ each in PBS. Sections were then incubated with $1 \mathrm{ml}$ staining solution mixture $(10 \mu$ staining solution $A, 10 \mu l$ staining solution $B$, $930 \mu \mathrm{l}$ staining solution $\mathrm{C}$ and $50 \mu \mathrm{l}$-gal solution) for $2 \mathrm{~h}$ at $37^{\circ} \mathrm{C}$. Following 3 washes with PBS, 5 fields of view from each of the 3 sections from each lung sample were examined using a light microscope (Olympus Corporation).

\section{Transmission electron microscopy (TEM)}

Murine lung tissues were fixed in $2 \%$ glutaraldehyde for at least $24 \mathrm{~h}$. Tissues were then immersed in $2 \%$ osmium tetroxide and $1 \%$ aqueous uranyl acetate, each for $1 \mathrm{~h}$. After washed with a series of ethanol solutions (50\%, $70 \%, 90 \%$ and $100 \%$ ), tissues were transferred to propylene oxide, incubated in a 1:1 mixture of propylene oxide and EMbed 812 (Electron Microscopy Sciences) for 1 hour and then placed in a $70^{\circ} \mathrm{C}$ oven to polymerize. Sections $(75-80 \mathrm{~nm})$ were cut using a Leica ultramicrotome equipped with a Diatome diamond knife and collected on 200-mesh copper grids. After poststained in $5 \%$ uranyl acetate for 10 min and in Reynold's lead citrate for 5

min, sections were observed using a 40-120 kV transmission electron microscope (FEI TECNAI G2 Spirit Biotwin, Hong Kong, China). For in vitro study, After treatment, HBEC were fixed with $2 \%$ glutaraldehyde/

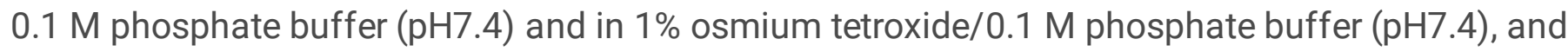
dehydrated with a graded series of ethanol. The operations subsequent were performed as described above.

\section{Immunofluorescence straining}


Murine lung frozen sections were fixed with $4 \%$ paraformaldehyde. After washing with PBS for several times and incubating with goat serum for $1 \mathrm{~h}$, they were incubated overnight with LC3B antibody (1:100,Cell Signaling Technology, USA)at $4{ }^{\circ} \mathrm{C}$. HBEC expressing EGFP-LC3B grown on 6-well culture slides were fixed with $4 \%$ paraformaldehyde for $15 \mathrm{~min}$ followed by permeabilization with $0.03 \%$ Triton X-100(Wako,16024751) for 60 min. After blocking with 0.1\%BSA(Sigma Aldrich,A2153) for 60 min, the primary and secondary antibodies were applied according to the manufacture's instructions. Confocal laser scanning microscopy analysis of mitochondria was performed by TOMM20 staining, assessed with a confocal microscope(Carl Zeiss LSM510,Tokyo,Japan).Mito Tracker Red CMX Ros (MTR; Molecular Probes-Life Technologies,M-7512) staining $\left(200 \mathrm{nM}, 30 \mathrm{~min}\right.$ at $\left.37^{\circ} \mathrm{C}\right)$ was also performed to evaluate the integrity of the mitochondrial membrane potential.

\section{Western blotting}

After treatments, murine lung tissues and HBEC were collected, digested and analyzed using a BCA assay (for protein concentration, Thermo Fisher Scientific,89900) with protease inhibitor cocktail(Roche Diagnostics, 11697498001). For each group, equal amounts of total protein were separated using SDSPAGE gels, transferred to polyvinylidene difluoride membrane(PVDF,Millipore,ISEQ00010), and then incubated overnight at $4^{\circ} \mathrm{C}$ with special primary antibody. Antibody used by mouse anti-Sirt1(Cell Signaling Technology,2947), rabbit anti-LC3B(Cell Signaling Technology,2577), rabbit anti-PINK1 (Abcam, ab23707), mouse anti-ACTB (Sigma-Aldrich, A5316), Foxo3A(Cell Signaling Technology, 2132), AcFoxo3a (Santa Cruz Biotechnology, sc-468). Then blots were incubated by anti-rabbit IgG, HRP-linked secondary antibody (1:5000,Cell Signaling Technology,7074) or anti-mouse IgG, HRP-linked secondary antibody (1:5000, Cell Signaling Technology, 7076) for $1 \mathrm{~h}$. Finally, Blots were scanned and detected by the luminescence method. Band intensity was analyzed using the Image $\mathrm{J}$ software(Version 1.45).

\section{Statistical analysis}

All the experimental data are presented as the means \pm SEM and analyzed by Prism version 6.0 (GraphPad Software, San Diego, USA). The $t$-test was performed to measure the differences between the two groups and one-way analysis of variance (ANOVA) followed by a Dunnett's test was performed to compare the differences among three or more groups. $P$-values $<0.05$ were considered statistical significance.

\section{Results}

\section{Effect of Sirt1 inhibitor on CSE induced changs in lung function}

Expression of Sirt1 in mouse with selisistat treatment was confirmed using western blot analysis. Our result showed that Sirt1 protein expression was drastically decreased in selisistat treatment mouse lung exposed to CS compare with their WT littermates (Fig.1A). Lung morphometry to examine alveolar mean linear intercept $\left(\mathrm{L}_{\mathrm{m}}\right)$.CS exposure for 6 months induced a modest airspace enlargement in WT mice, whereas SIRT1 deficient mice exhibit airspace enlargement (Fig.1B). To evaluate the effect of Sirt1 
inhibitor on CSE induced changes in lung function, Lung mechanical properties was employed to evaluate lung compliance and $\mathrm{R}_{\mathrm{L}}$ at $3 \mathrm{cmH}_{2} \mathrm{O}$ positive end-expiratory pressure were obtained by fitting a model to each impedance spectrum. Our data showed that lung compliance was augmented in WT mice exposed to CS for 6 months, the effect of which was accentuated in selisistat treatment mice (Fig.1C and D). Sirt1 deficiency decreased total lung resistance $\left(R_{L}\right)$, although no significant change in $R_{L}$ in WT mice was observed after 6 months of CS exposure.

\section{Effect of Sirt1 inhibitor on CSE-induced airspace enlargement, cellular senescence and mitochondrial morphology}

To evaluate the effect of Sirt1 inhibitor on CSE induced lung injury, SA- $\beta$-gal activity assay and transmission electron microscopy were used to examine cellular senescence and mitochondrial morphology, respectively. Our finding revealed that CS exposure for 6 months induced a modest airspace enlargement in WT mice, which was further augmented in selisistat treated mice. As expected, CS exposure significantly the level of SAßgal activity in lungs of selisistat treated mice versus WT littermates. Transmission electron microscopy also revealed more swollen an damaged mitochondria in WT mice exposed to CS, the effect of which was augmented by Sirt1 inhibitor treated mice. The mitochodria were slightly changed in selisistat treated mice compared with WT mice. (Fig2)

\section{Effect of Sirt1 inhibitor on CSE-induced autophagy regulation}

To determine the effect of Sirt1 inhibitor on CES induced autophagy regulation, immunofluorescence and western blotting were used to examine LC3 puncta in frozen section of lungs and LC3B protein expression, respectively. Our data revealed that the number of LC3B puncta was significant decreased in WT mice exposed to CS, with a more pronounced response in Sirt1 inhibitor mice(Fig3A).Meanwhile western blot analysis revealed that the LC3 $₫$ protein level were overtly downregulated in WT mice exposed to CS, the effect of which was accentuated by Sirt1 inhibitor(Fig3B).Sirt1 deficiency itself elicited a decrease in LC3 puncta and LC3区 level.

\section{Signaling mechanism(s) in Sirt1 inhibitor induced autophagy regulation in emphysema}

To explore the possible mechanism(s) in Sirt1 inhibitor accentuation of CSE induces changes in autophagy, western blot analysis was employed to examine the level of Ac-Foxo3A and Pink1. our result revealed that the ratio of Ac-Foxo3a to Foxo3A was greatly increased following CSE exposure, the effect of which was exacerbated by more upregulated by Sirt1 inhibitor. Sirt1 deficiency also increased the ratio of Ac-Foxo3a to Foxo3a. PINK1 expression was reduced in WT mice exposed to CSE, the effect of which was unaffected by Sirt1 inhibitor.Sirt1 deficiency also decreased the level of PINK1.(Fig.4.)

\section{Alteration of levels of Sirt1 and LC3B by CSE in primary human bronchial epithelial cells}

HBEC were treated with $10 \%$ fetal calf serum and CSE (1\%) in the presence or absence of SRT1720 for $48 \mathrm{~h}$, and the control cells were cultured with $10 \%$ fetal calf serum. Western blotting revealed that LC3囚 
protein level began to decline and p62 expression began to rise at $\operatorname{CSE}(1 \%)$ and was further reduced at $\operatorname{CSE}(2.5 \%)$,consistent with the previous report[14].We next examined the time course of autophagy downregulation. HBEC were exposed to CS $(2.5 \%)$ for $6-48$ hour, while $10 \%$ fetal calf serum were used as control. A significant reduction of LC3\ protein levels and increased p62 expression was observed from $24 \mathrm{~h}$,the effect of which was present throughout the entire examination period. The expression of Sirt1 began to decrease at $12 \mathrm{~h}$ and remained consistent thereafter(Fig5).

\section{Effect of Sirt1 overexpression on CSE induced ROS production, apoptosis, and mitophagy in HBEC}

To determine the effect of Sirt1 overexpression on CSE induced the reduction of mitochondrial ROS production, DCFH-DA assays and MitoSOX Red staining were applied to evaluate the association between mitophagy and ROS production in HBEC. Our data showed that CSE treatment results in a rise of damaged mitochondria, total and mitochondrial ROS production, which was significantly reduced by SRT1720(Fig6A,B). JC-1 staining revealed that mitochondrial membrane potential of CSE group was significantly decreased, compared with normal control group, the effect of which was largely counteracted by SRT1720(Fig6C). Result from transmission electron microscopy showed that more obviously swollen and damaged mitochondria were observed in cells with CSE treatment, with were reserved by SRT1720(Fig6D).

\section{Effect of Sirt1 overexpression on CSE induced autophagy regulation}

To examine the effect of Sirt1 overexpression on CSE induced autophagy regulation, confocal microscopy was performed in HBEC stably experssion EGFP-LC3. Colocalization of TOMM20-stained mitochondria and EGFP-LC3B dots was used to determine autophagosome formation,and western blotting determining LC3B and p62 protein level was used.Our results showed that although CSE induced EGFP-LC3B dot formation,colocalization with mitochondria was barely detected in the absence of SRT1720. SRT1720 treatment alone induced EGFG-LC3B dot formation accompanied by limited colocalization with TOMM20-stained mitochondria(yellow dots),CSE treatment markedly enhanced EGFPLC3B dot formation concomitant colocalization with TOMM20-stained mitochondria in the presence of SRT1720(Fig).For cellular exposed to CSE,the level of LC3 $\$ were with significantly difference after overexpressing of Sirt1. The upregulated expression of p62 in response to CSE challenge was reversed by SRT1720.

\section{Effect of Sirt1 overexpression on the mechanism(s) involved in CSE induced autophagy regulation}

To determine the effect of Sirt1 overexpression on the mechanism(s) involved in CSE induced autophagy regulation,western blotting analysis was applied to detect the expression of Ac-Foxo3A and Pink1. The results revealed that Sirt1 level was increased after SRT1720 treatment. In CSE condition ,Sirt1 and Pink1 were both reduced,while the ratio of Ac-Foxo3A to Foxo3a was increased.The effect of CSE treatment was obviously reversed by overexpressing Sirt 1 and this effect was also noted in normal HBEC with SRT1720. 


\section{Discussion}

Our study finding that CSE induced HBEC and COPD downregulates anti-aging sirt1 and suppressed the Sirt1-Foxo3A signaling cascade. It is the protein levels demonstrated decreased Pink1 in CSE group compared with control group. Concomitantly, CSE induced fragmented mitochondria accumulated and slightly mitophagy activation[27, 28]. Activation of Sirt1 mediated Foxo3 deacetylation, resulting in an increase of Pink1 in HBEC treated with CSE, restoration of HBEC autophagy and protection against HBEC apoptosis[29, 30]. SIRT1 inhibitor lead to suppressed Sirt1-Foxo3 signaling and Pink1 mediated mitophagy may participate in CSE induced downregulation of HBEC autophagy and increased HBEC apoptosis[31]. SRT1720 and selisistat experiments clarify that both Sirt1 and Foxo3 are indicate a likely role for mitophagy in the pathogenesis of CSE exposure[32-34]. Moreover, recent studies observed modestly upregulation of PINK1 in lung homogenates from COPD patients[35, 36]. Therefore, the exact role of PINK1 in mitophagy has not been well done defined because of the existing contradistinction.

By mitophagic eliminate of damaged mitochondria and damaged organelles within lysosome, autophagy maintains cellular homeostasis[37]. Improper mitophagic has been implicated in the pathogenesis to different types of pulmonary diseases, and recently papers finding insufficient autophagy associated with complications of pulmonary including acute lung injury and idiopathic pulmonary fibrosis[38]. The depressed autophagy was proved to have a detrimental role in lung function of CES induced COPD although contrary findings have also been reported[39]. To elucidate the function role of mitophagy that using activation and inhibitor experiments in vitro and in vivo models. The CSE induced cellular damage was attenuated with Sirt1 overexpression, while the cellular damage was exacerbated in emphysema mice deficient in Sirt1, suggesting an insufficient role for mitophagy in COPD/emphysema[14, 40].

In the line with the recent findings CSE-induced emphysema lung dysfunction, the Sirt1-Foxo3-Pink1 pathway is involved in regulating the mitophagic elimination of CSE induced damaged mitochondria that modulates cellular senescence[41]. In this research, EM shows autophagy and mitophagy activation induced by Sirt1 result in attenuated mitochondrial numbers and enhanced degradation of mitochondrial debris(Fig 2).It has been reported that Pink1 levels in COPD smokers tend to be lower than those in control lung, and Pink1 overexpression increase mitophagy activity, indication that Pink1 is not only a pivotal predisposition to COPD development but also a crucial role in mitochondrial quality control in terms of regulation of senescence associated disorders[42, 43]. In this work, we found suppressed Pink1 in CSE induced HBEC or deficiency of Sirt1 condition, in concordance with other papers. Some studies demonstrated an inhibited autophagic flux in COPD lungs. In our vivo experiment, autophagy and Pink1 mediated mitophagy both reduced 6 months after CSE exposure, which can be regard as advanced stages of COPD.

Sirt1, as one of the intracellular deacetylases, is an important role in many processes of mitochondrial function[13]. Intriguingly, Studies demonstrated that Sirt1 may act as a tumor suppressor in several types of cancers. In response to CSE stimulus, Sirt1 was deficiency is increased apoptotic and mitochondrial dysfunction in bronchial epithelial cell. Airspace were enlarged obviously and the amount of alveoli was 
significantly decrease in selisistat treat mice than control group mice, along with more depressed mitophagy. Sirt1 protects against emphysema through Foxo3-mediated maintain mitochondrial homeostasis and reduction of cellular senescence. Overexpression of Sirt1 inverted Sirt1-Foxo3a-Pink1 signaling mediated mitophagy and senescence of epithelial cell, indicating that Sirt1-Foxo3a-Pink1 signaling was a crucial pathway involved in overexpression Sirt1 promotion of alveoli survival under CSE exposure[11, 44, 45]. Recent advances demonstrated inflammatory reactions involved in Sirt1 induced regulation of mitophagic degradation[46]. It was reported that Sirt1 activated mitophagy by inducing TLR9(toll-like receptor 9) recognition and activation of IL1B/interleukin 1及,via deacetylation Foxo3 with the subsequent downregulation of NALP3 inflammasome[47, 48].

\section{Conclusion}

In summary, We demonstrated that the CSE induced mitophagy and autophagy by Sirt1-Foxo3-Pink1 path in HBEC. Suppressed Sirt1-Foxo3-Pink1 signaling and reduced Pink1 expression levels in COPD lung that downregulation of mitophagy and acceleration of cell senescence. Although the precise mechanism through other positive regulators regulated epithelial cell mitophagy in the setting of CSE exposure is still unclear. Our current study further supported the notion that the effect of Sirt1-Foxo3 signaling and Pink1association mitophagy may explain the mechanism of insufficient mitophagy in COPD lung.

\section{Abbreviations}

ANOVA: One-way analysis of variance; COPD: Chronic obstructive pulmonary disease; CS: Cigarette smoke; CSE: Cigarette smoke extract; Foxo3: Forkhead-box class 03; HBEC: Human bronchial epithelial cells; PINK1: phosphatase/tensin homolog on chromosome 10 (PTEN)-induced putative kinase 1; ROS: Reactive oxygen species; TMP: Total particulate matter; TEM: Transmission electron microscopy; WB: Western blot;

\section{Declarations}

\section{Acknowledgements}

We thank professor Yaqing Li for his guidance on the writing of this paper.

\section{Authors' contributions}

DY and YJ conceived of the study. HJ and YL participated in design of the study and performed the experiments. HJ carried out the statistical analysis. DY helped in the interpretation of the data and coordination and drafted the manuscript. All authors read and approved the final manuscript.

\section{Funding}

This work was funded by the National Natural Science Foundation of China 
(No. 8187010048).

Availability of data and materials

The software and all relevant raw data are freely available to scientists.

Declarations

Ethics approval and consent to participate

All animal experiments were approved by the Experimental Animal Ethics Committee of Hangzhou medical college and performed in accordance with the guidelines for the care and use of laboratory animals set by Zhejiang Industry University (Hangzhou,China).

Consent for publication

Not applicable.

Competing interests

The authors declare that they have no competing interests.

\section{References}

1. Ehteshami-Afshar S, FitzGerald JM, Doyle-Waters MM, Sadatsafavi M: The global economic burden of asthma and chronic obstructive pulmonary disease.Int J Tuberc Lung Dis 2016, 20:11-23.

2. Jiang $Y$, Wang $X$, Hu D: Mitochondrial alterations during oxidative stress in chronic obstructive pulmonary disease.International Journal of Chronic Obstructive Pulmonary Disease 2017, Volume 12:1153-1162.

3. Wiegman CH, Michaeloudes C, Haji G, Narang P, Clarke CJ, Russell KE, Bao W, Pavlidis S, Barnes PJ, Kanerva J, et al: Oxidative stress-induced mitochondrial dysfunction drives inflammation and airway smooth muscle remodeling in patients with chronic obstructive pulmonary disease.Journal of Allergy and Clinical Immunology 2015, 136:769-780.

4. Konokhova Y, Spendiff S, Jagoe RT, Aare S, Kapchinsky S, MacMillan NJ, Rozakis P, Picard M, Aubertin-Leheudre M, Pion $\mathrm{CH}$, et al: Failed upregulation of TFAM protein and mitochondrial DNA in oxidatively deficient fibers of chronic obstructive pulmonary disease locomotor muscle.Skeletal Muscle 2016, 6.

5. Yoon CM, Nam M, Oh Y-M, Dela Cruz CS, Kang M-J: Mitochondrial Regulation of Inflammasome Activation in Chronic Obstructive Pulmonary Disease.Journal of Innate Immunity 2016, 8:121-128.

6. Leermakers PA, Schols AMWJ, Kneppers AEM, Kelders MCJM, de Theije CC, Lainscak M, Gosker HR: Molecular signalling towards mitochondrial breakdown is enhanced in skeletal muscle of patients with chronic obstructive pulmonary disease (COPD).Scientific Reports 2018, 8. 
7. Karimova A, Oltulu YM, Azaklı H, Kara M, Ustek D, Tutluoglu B, Onaran I: Lack of association between increased mitochondrial DNA4977 deletion and ATP levels of sputum cells from chronic obstructive pulmonary disease patients versus healthy smokers.Mitochondrial DNA Part A 2015, 28:361-369.

8. Wen W, Yu G, Liu W, Gu L, Chu J, Zhou X, Liu Y, Lai G: Silencing FUNDC1 alleviates chronic obstructive pulmonary disease by inhibiting mitochondrial autophagy and bronchial epithelium cell apoptosis under hypoxic environment.Journal of Cellular Biochemistry 2019, 120:17602-17615.

9. Haji G, Wiegman CH, Michaeloudes C, Patel MS, Curtis K, Bhavsar P, Polkey MI, Adcock IM, Chung KF: Mitochondrial dysfunction in airways and quadriceps muscle of patients with chronic obstructive pulmonary disease.Respiratory Research 2020, 21.

10. Fujii S, Hara H, Araya J, Takasaka N, Kojima J, Ito S, Minagawa S, Yumino Y, Ishikawa T, Numata T, et al: Insufficient autophagy promotes bronchial epithelial cell senescence in chronic obstructive pulmonary disease. Oncolmmunology 2014, 1:630-641.

11. Rajendrasozhan S, Yang S-R, Kinnula VL, Rahman I: SIRT1, an Antiinflammatory and Antiaging Protein, Is Decreased in Lungs of Patients with Chronic Obstructive Pulmonary Disease.American Journal of Respiratory and Critical Care Medicine 2008, 177:861-870.

12. Louvet L, Leterme D, Delplace S, Miellot F, Marchandise P, Gauthier V, Hardouin P, Chauveau C, Ghali Mhenni 0 : Sirtuin 1 deficiency decreases bone mass and increases bone marrow adiposity in a mouse model of chronic energy deficiency.Bone 2020, 136.

13. Jia Y, Zheng Z, Wang Y, Zhou Q, Cai W, Jia W, Yang L, Dong M, Zhu X, Su L, Hu D: SIRT1 Is a Regulator in High Glucose-Induced Inflammatory Response in RAW264.7 Cells.Plos One 2015, 10.

14. Yao H, Chung S, Hwang J-w, Rajendrasozhan S, Sundar IK, Dean DA, McBurney MW, Guarente L, Gu W, Rönty M, et al: SIRT1 protects against emphysema via FOXO3-mediated reduction of premature senescence in mice.Journal of Clinical Investigation 2012, 122:2032-2045.

15. Li W, Du M, Wang Q, Ma X, Wu L, Guo F, Ji H, Huang F, Qin G: Fox01 Promotes Mitophagy in the Podocytes of Diabetic Male Mice via the PINK1/Parkin Pathway.Endocrinology 2017, 158:21552167.

16. Shin NR, Ko JW, Kim JC, Park G, Kim SH, Kim MS, Kim JS, Shin IS: Role of melatonin as an SIRT1 enhancer in chronic obstructive pulmonary disease induced by cigarette smoke.Journal of Cellular and Molecular Medicine 2020, 24:1151-1156.

17. Gu C, Li Y, Liu J, Ying X, Liu Y, Yan J, Chen C, Zhou H, Cao L, Ma Y: LncRNA-mediated SIRT1/FoxO3a and SIRT1/p53 signaling pathways regulate type II alveolar epithelial cell senescence in patients with chronic obstructive pulmonary disease.Molecular Medicine Reports 2017, 15:3129-3134.

18. Yao Z-q, Zhang X, Zhen Y, He X-Y, Zhao S, Li X-F, Yang B, Gao F, Guo F-Y, Fu L, et al: A novel smallmolecule activator of Sirtuin-1 induces autophagic cell death/mitophagy as a potential therapeutic strategy in glioblastoma.Cell Death \& Disease 2018, 9.

19. Gu C, Li Y, Xu W-L, Yan J-P, Xia Y-j, Ma Y-Y, Chen C, Wang H-J, Tao H-q: Sirtuin 1 Activator SRT1720 Protects Against Lung Injury via Reduction of Type II Alveolar Epithelial Cells Apoptosis in Emphysema.COPD: Journal of Chronic Obstructive Pulmonary Disease 2014, 12:444-452. 
20. Galli U, Mesenzani O, Coppo C, Sorba G, Canonico PL, Tron GC, Genazzani AA: Identification of a sirtuin 3 inhibitor that displays selectivity over sirtuin 1 and 2.European Journal of Medicinal Chemistry 2012, 55:58-66.

21. Das S, Mitrovsky G, Vasanthi HR, Das DK: Antiaging Properties of a Grape-Derived Antioxidant Are Regulated by Mitochondrial Balance of Fusion and Fission Leading to Mitophagy Triggered by a Signaling Network of Sirt1-Sirt3-Foxo3-PINK1-PARKIN.Oxidative Medicine and Cellular Longevity 2014, 2014:1-13.

22. Yao H, Edirisinghe I, Rajendrasozhan S, Yang SR, Caito S, Adenuga D, Rahman I: Cigarette smokemediated inflammatory and oxidative responses are strain-dependent in mice.Am J Physiol Lung Cell Mol Physiol 2008, 294:L1174-1186.

23. Foronjy RF, Mirochnitchenko O, Propokenko O, Lemaitre V, Jia Y, Inouye M, Okada Y, D'Armiento JM: Superoxide dismutase expression attenuates cigarette smoke or elastase-generated emphysema in mice.Am J Respir Crit Care Med 2006, 173:623-631.

24. Araya J, Cambier S, Markovics JA, Wolters P, Jablons D, Hill A, Finkbeiner W, Jones K, Broaddus VC, Sheppard D, et al: Squamous metaplasia amplifies pathologic epithelial-mesenchymal interactions in COPD patients.J Clin Invest 2007, 117:3551-3562.

25. Yao H, Arunachalam G, Hwang Jw, Chung S, Sundar IK, Kinnula VL, Crapo JD, Rahman I: Extracellular superoxide dismutase protects against pulmonary emphysema by attenuating oxidative fragmentation of ECM.Proceedings of the National Academy of Sciences 2010, 107:15571-15576.

26. Yao H, Yang SR, Edirisinghe I, Rajendrasozhan S, Caito S, Adenuga D, O'Reilly MA, Rahman I: Disruption of p21 attenuates lung inflammation induced by cigarette smoke, LPS, and fMLP in mice.Am J Respir Cell Mol Biol 2008, 39:7-18.

27. Hara H, Araya J, Ito S, Kobayashi K, Takasaka N, Yoshii Y, Wakui H, Kojima J, Shimizu K, Numata T, et al: Mitochondrial fragmentation in cigarette smoke-induced bronchial epithelial cell senescence.Am J Physiol Lung Cell Mol Physiol 2013, 305:L737-746.

28. Diot A, Morten K, Poulton J: Mitophagy plays a central role in mitochondrial ageing.Mamm Genome 2016, 27:381-395.

29. Di Vincenzo S, Heijink IH, Noordhoek JA, Cipollina C, Siena L, Bruno A, Ferraro M, Postma DS, Gjomarkaj M, Pace E: SIRT1/FoxO3 axis alteration leads to aberrant immune responses in bronchial epithelial cells.J Cell Mol Med 2018, 22:2272-2282.

30. Tang YL, Zhou Y, Wang YP, He YH, Ding JC, Li Y, Wang CL: Ginsenoside Rg1 protects against Sca-1(+) HSC/HPC cell aging by regulating the SIRT1-FOXO3 and SIRT3-SOD2 signaling pathways in a gamma-ray irradiation-induced aging mice model.Exp Ther Med 2020, 20:1245-1252.

31. Kim H, Baek CH, Chang JW, Yang WS, Lee SK: Febuxostat, a novel inhibitor of xanthine oxidase, reduces ER stress through upregulation of SIRT1-AMPK-HO-1/thioredoxin expression.Clin Exp Nephrol 2020, 24:205-215.

32. Zhang F, Li ZL, Xu XM, Hu Y, Yao JH, Xu W, Jing HR, Wang S, Ning SL, Tian XF: Protective effects of icariin-mediated SIRT1/FOXO3 signaling pathway on intestinal ischemia/reperfusion-induced acute 
lung injury.Mol Med Rep 2015, 11:269-276.

33. Kume S, Uzu T, Horiike K, Chin-Kanasaki M, Isshiki K, Araki S, Sugimoto T, Haneda M, Kashiwagi A, Koya D: Calorie restriction enhances cell adaptation to hypoxia through Sirt1-dependent mitochondrial autophagy in mouse aged kidney.J Clin Invest 2010, 120:1043-1055.

34. Pace E, Di Vincenzo S, Ferraro M, Bruno A, Dino P, Bonsignore MR, Battaglia S, Saibene F, Lanata L, Gjomarkaj M: Carbocysteine counteracts the effects of cigarette smoke on cell growth and on the SIRT1/Fox03 axis in bronchial epithelial cells.Exp Geronto/ 2016, 81:119-128.

35. Ito S, Araya J, Kurita Y, Kobayashi K, Takasaka N, Yoshida M, Hara H, Minagawa S, Wakui H, Fujii S, et al: PARK2-mediated mitophagy is involved in regulation of HBEC senescence in COPD pathogenesis.Autophagy 2015, 11:547-559.

36. Gong G, Song M, Csordas G, Kelly DP, Matkovich SJ, Dorn GW, 2nd: Parkin-mediated mitophagy directs perinatal cardiac metabolic maturation in mice.Science 2015, 350:aad2459.

37. Zhai M, Li B, Duan W, Jing L, Zhang B, Zhang M, Yu L, Liu Z, Yu B, Ren K, et al: Melatonin ameliorates myocardial ischemia reperfusion injury through SIRT3-dependent regulation of oxidative stress and apoptosis.J Pineal Res 2017, 63.

38. Song YM, Lee YH, Kim JW, Ham DS, Kang ES, Cha BS, Lee HC, Lee BW: Metformin alleviates hepatosteatosis by restoring SIRT1-mediated autophagy induction via an AMP-activated protein kinase-independent pathway.Autophagy 2015, 11:46-59.

39. Li X, He S, Ma B: Autophagy and autophagy-related proteins in cancer.Mol Cancer 2020, 19:12.

40. Yu L, Liu X, Li X, Yuan Z, Yang H, Zhang L, Jiang Z: Protective effects of SRT1720 via the HNF1alpha/FXR signalling pathway and anti-inflammatory mechanisms in mice with estrogeninduced cholestatic liver injury. Toxicol Lett 2016, 264:1-11.

41. Li F, Fan X, Zhang Y, Zhang Y, Ma X, Kou J, Yu B: Inhibition of myosin IIA-actin interaction prevents ischemia/reperfusion induced cardiomyocytes apoptosis through modulating PINK1/Parkin pathway and mitochondrial fission. Int J Cardio/ 2018, 271:211-218.

42. Tsubouchi K, Araya J, Kuwano K: PINK1-PARK2-mediated mitophagy in COPD and IPF pathogeneses.Inflamm Regen 2018, 38:18.

43. Lin Q, Li S, Jiang N, Shao X, Zhang M, Jin H, Zhang Z, Shen J, Zhou Y, Zhou W, et al: PINK1-parkin pathway of mitophagy protects against contrast-induced acute kidney injury via decreasing mitochondrial ROS and NLRP3 inflammasome activation.Redox Biol 2019, 26:101254.

44. Lv W, Jiang J, Li Y, Fu L, Meng F, Li J: MiR-302a-3p aggravates myocardial ischemia-reperfusion injury by suppressing mitophagy via targeting FOX03.Exp Mol Pathol 2020, 117:104522.

45. Kim J, Fiesel FC, Belmonte KC, Hudec R, Wang WX, Kim C, Nelson PT, Springer W, Kim J: miR-27a and miR-27b regulate autophagic clearance of damaged mitochondria by targeting PTEN-induced putative kinase 1 (PINK1).Mol Neurodegener 2016, 11:55.

46. Birrell MA, Eltom S: The role of the NLRP3 inflammasome in the pathogenesis of airway disease.Pharmacol Ther 2011, 130:364-370. 
47. dos Santos G, Kutuzov MA, Ridge KM: The inflammasome in lung diseases.Am J Physiol Lung Cell Mol Physiol 2012, 303:L627-633.

48. Miura K, Kodama Y, Inokuchi S, Schnabl B, Aoyama T, Ohnishi H, Olefsky JM, Brenner DA, Seki E: Tolllike receptor 9 promotes steatohepatitis by induction of interleukin-1 beta in mice.Gastroenterology 2010, 139:323-334 e327.

Figures 
A
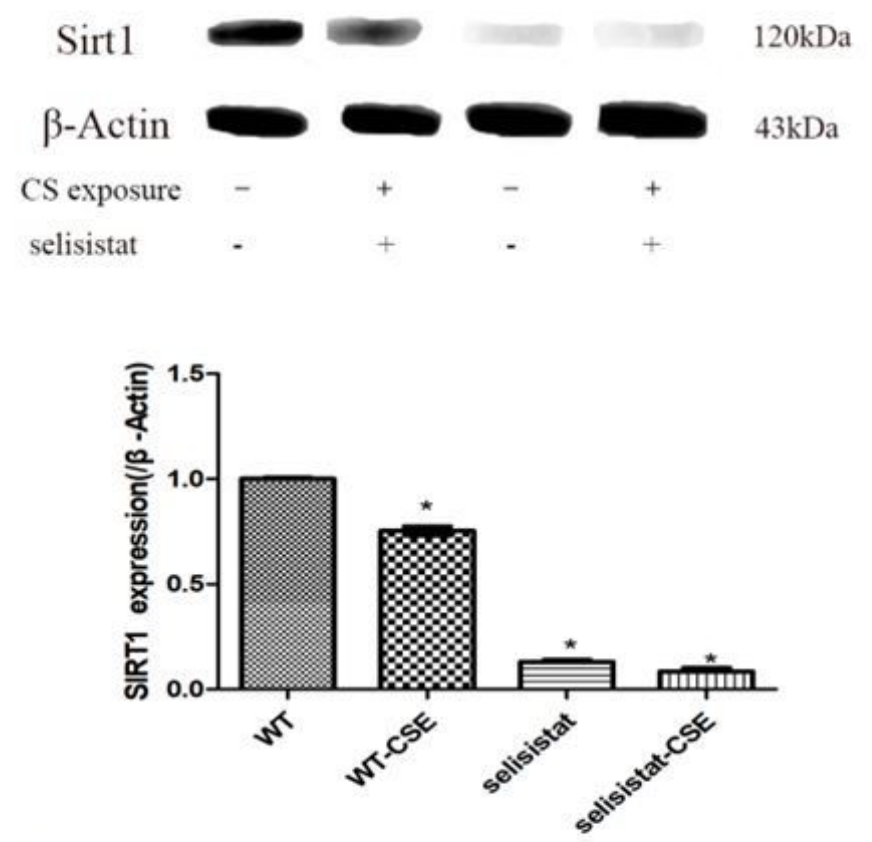

C

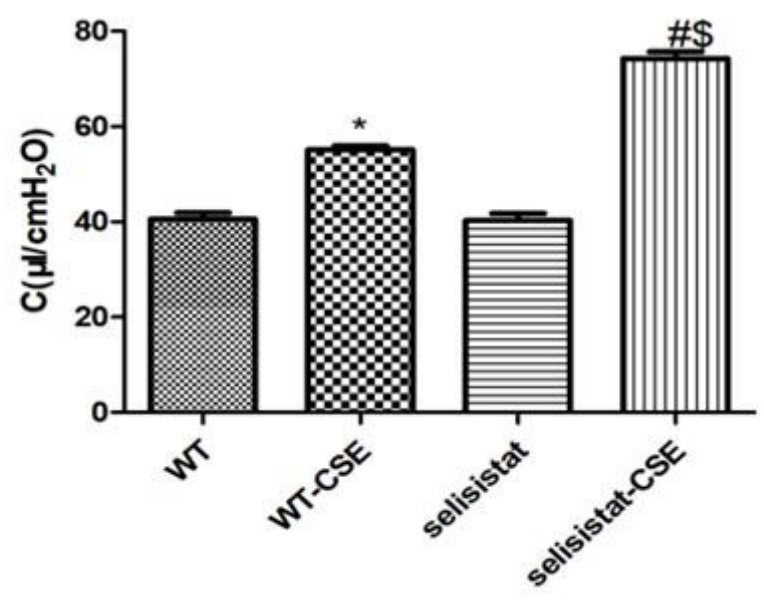

B

WT
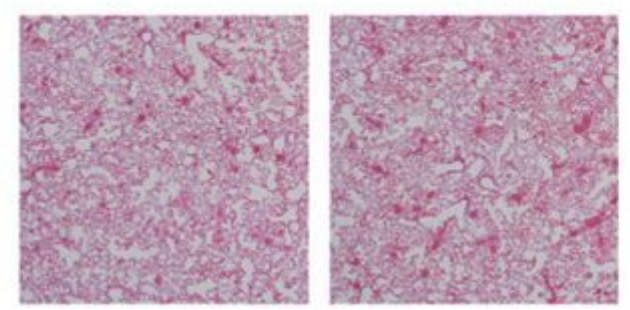

CS

exposure

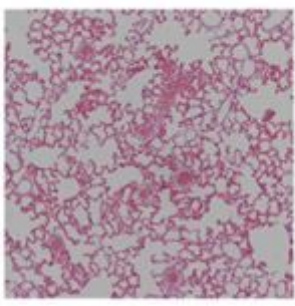

selisistat

(-)

(+)

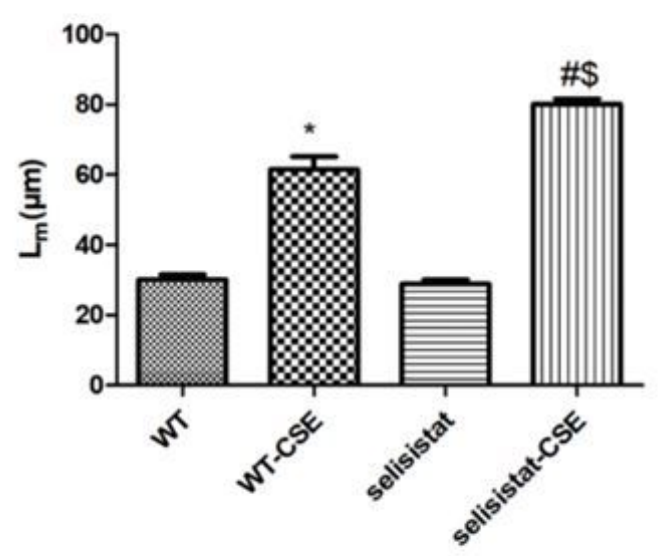

D

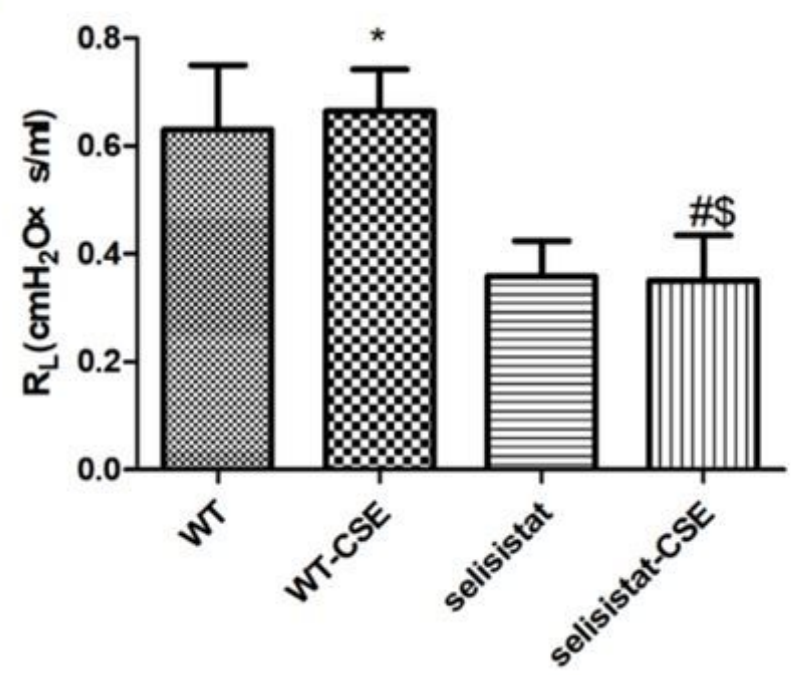

Figure 1

Please see the Manuscript file for the complete figure caption. 
A

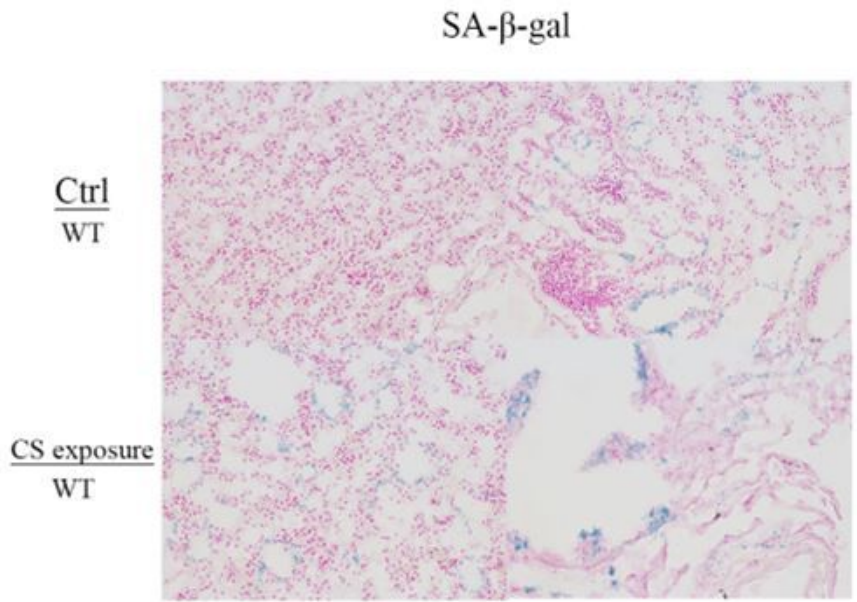

selisistat

(-)
WT

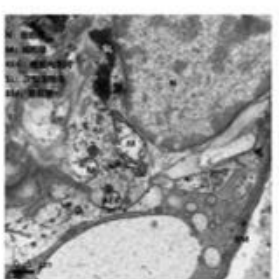

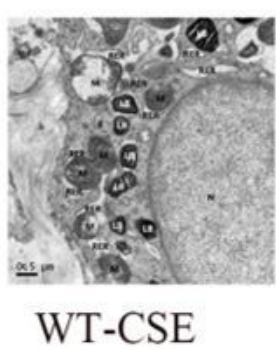

$(+)$

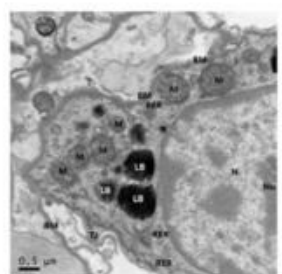

selisistat
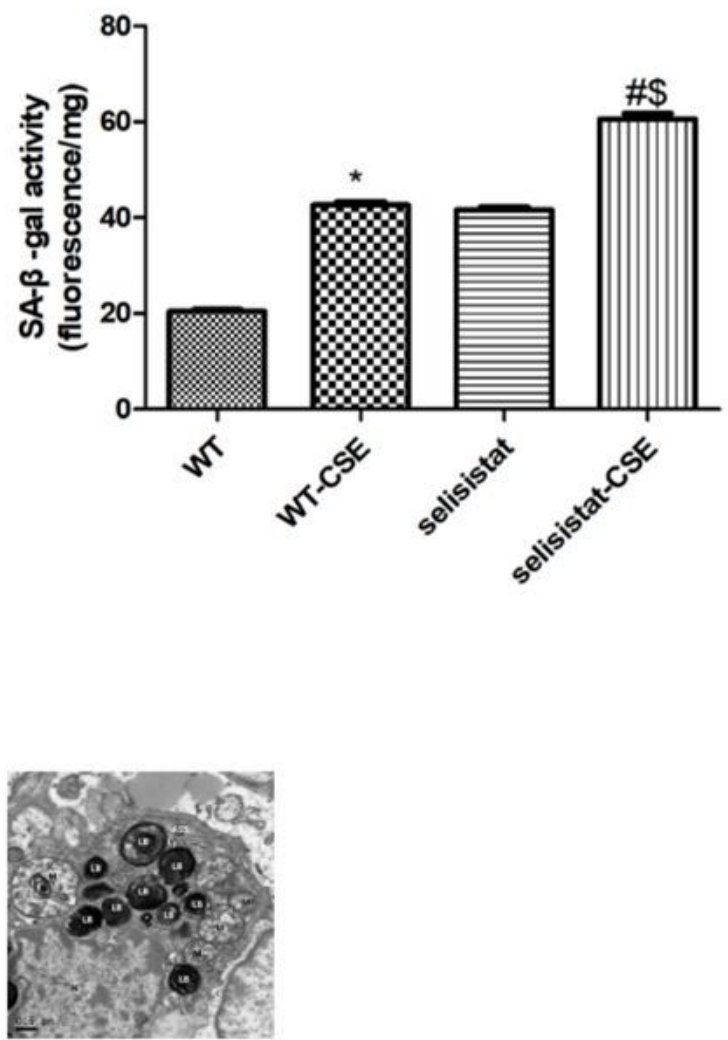

selisistat-CSE

Figure 2

Please see the Manuscript file for the complete figure caption. 
A
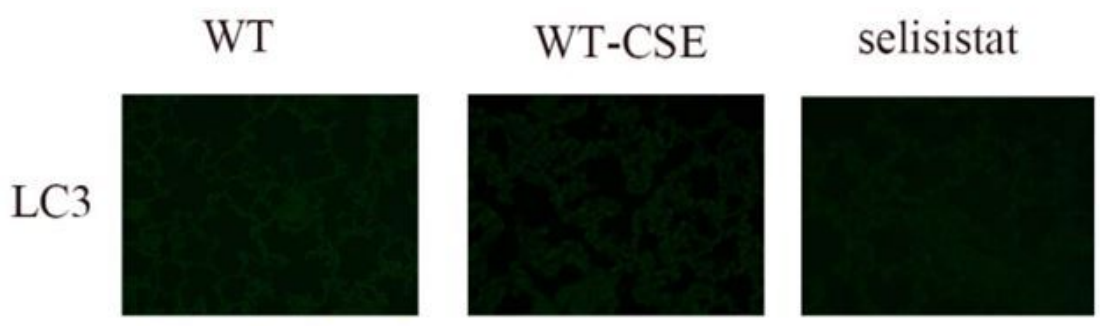

selisistat-CSE
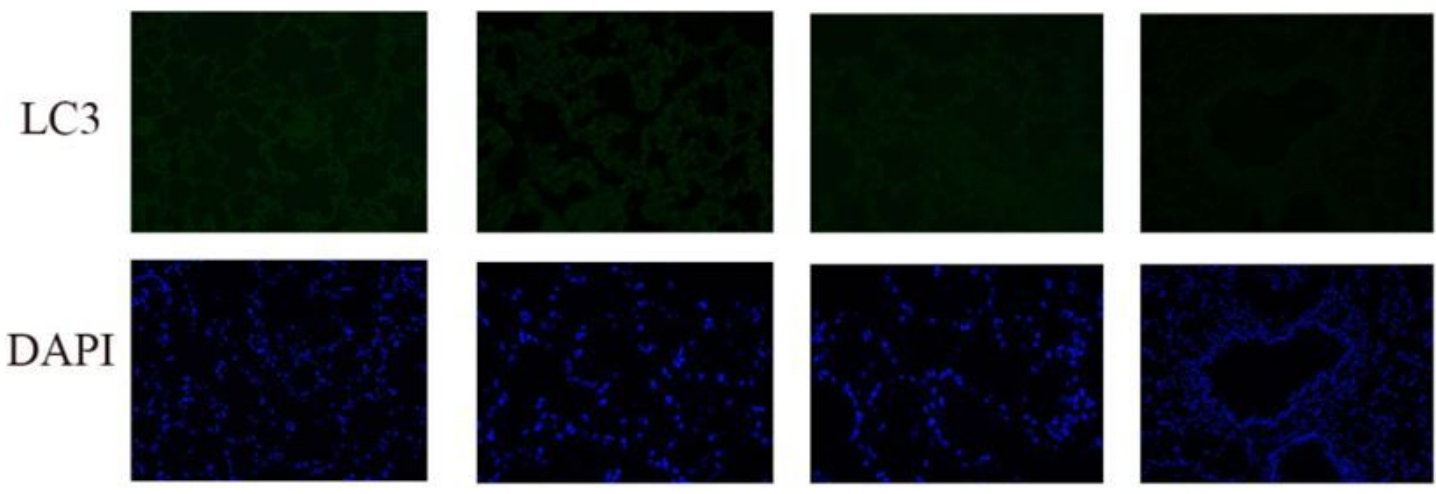

B

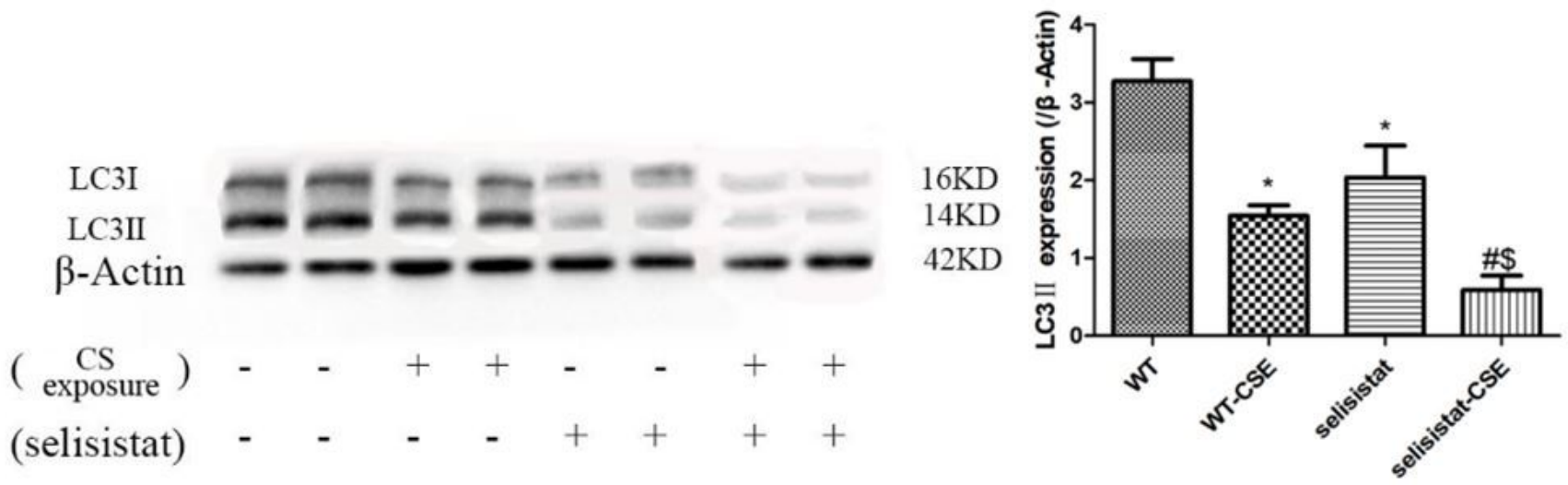

Figure 3

Please see the Manuscript file for the complete figure caption. 
A

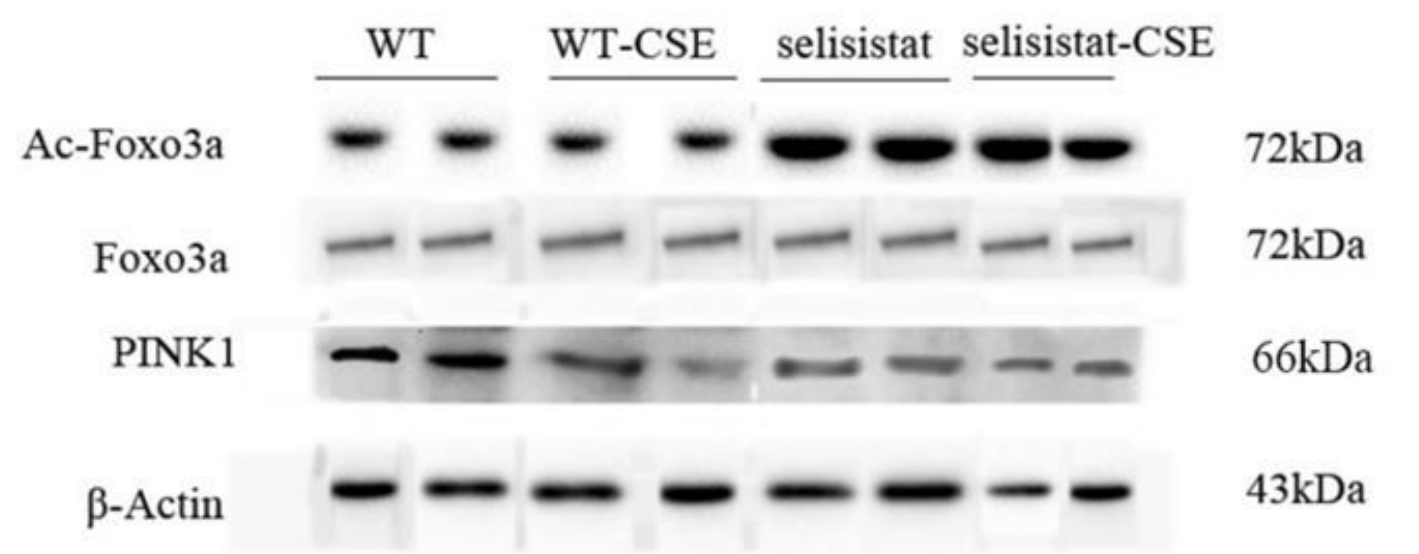

B

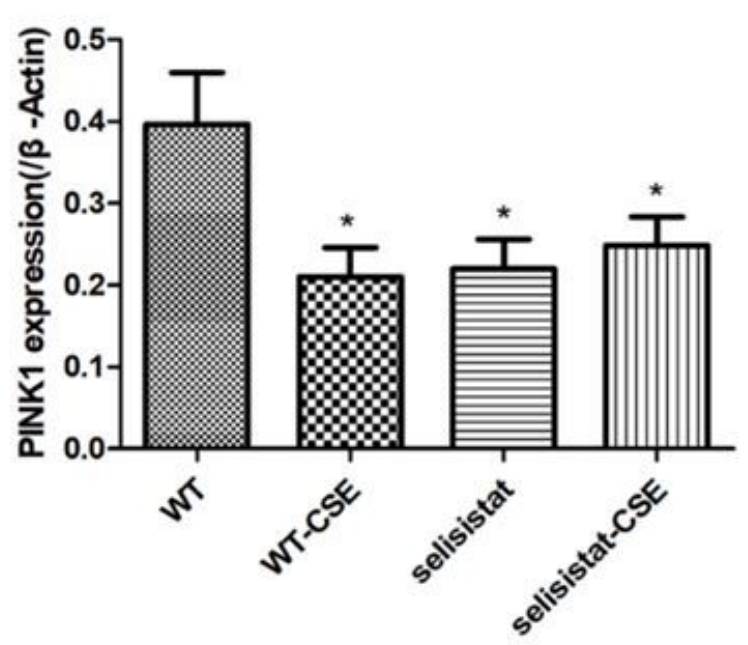

D

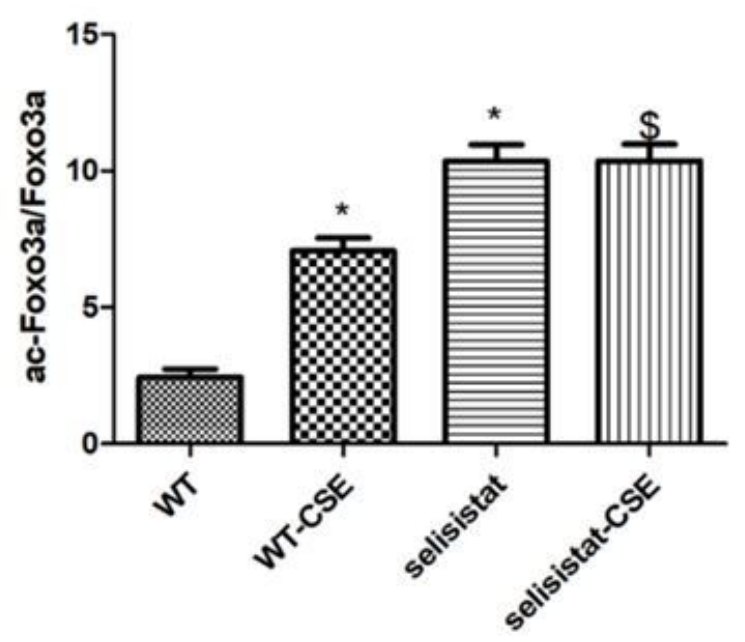

C

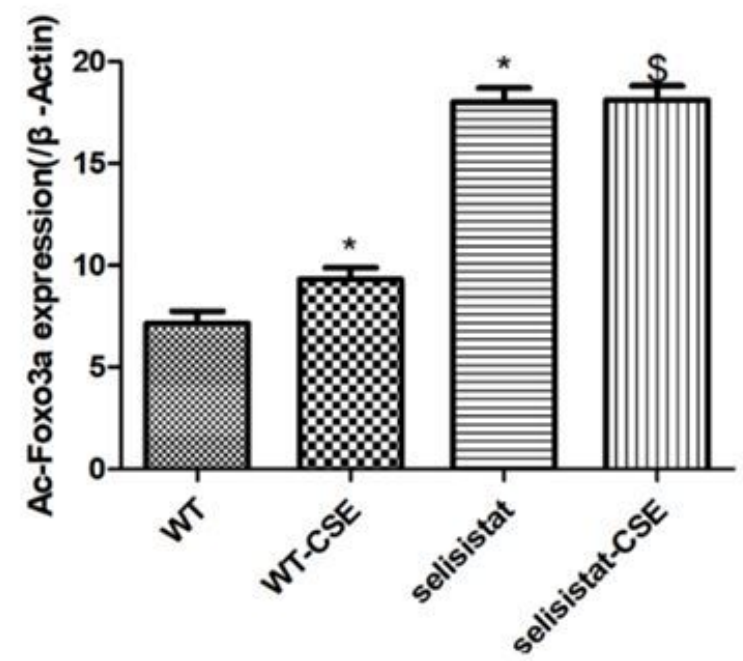

E

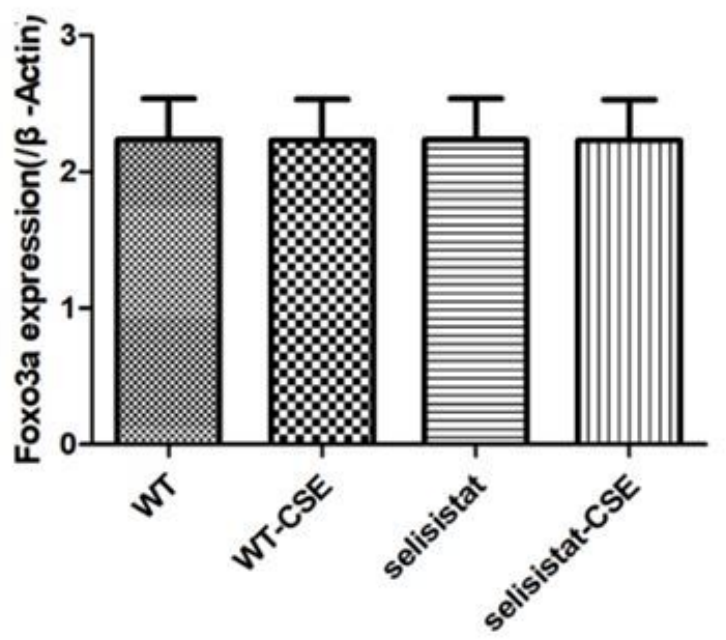

Figure 4

Please see the Manuscript file for the complete figure caption. 
A

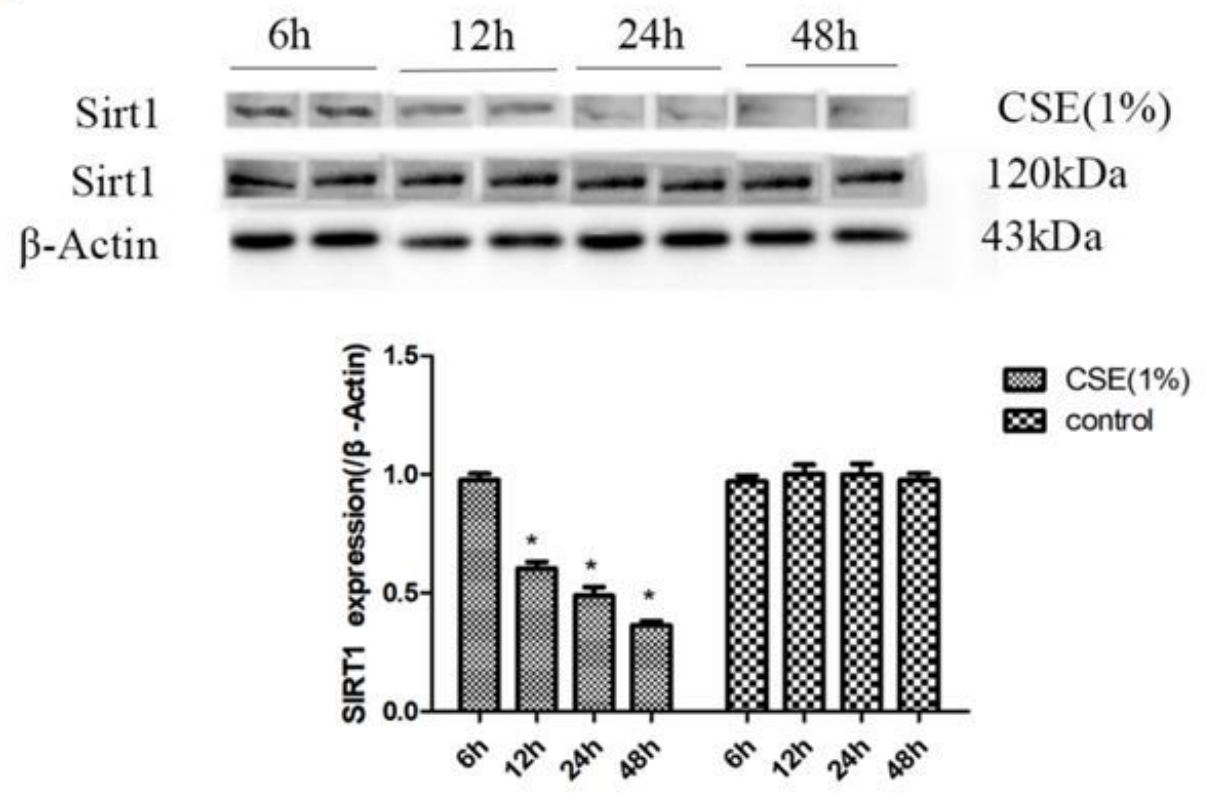

B
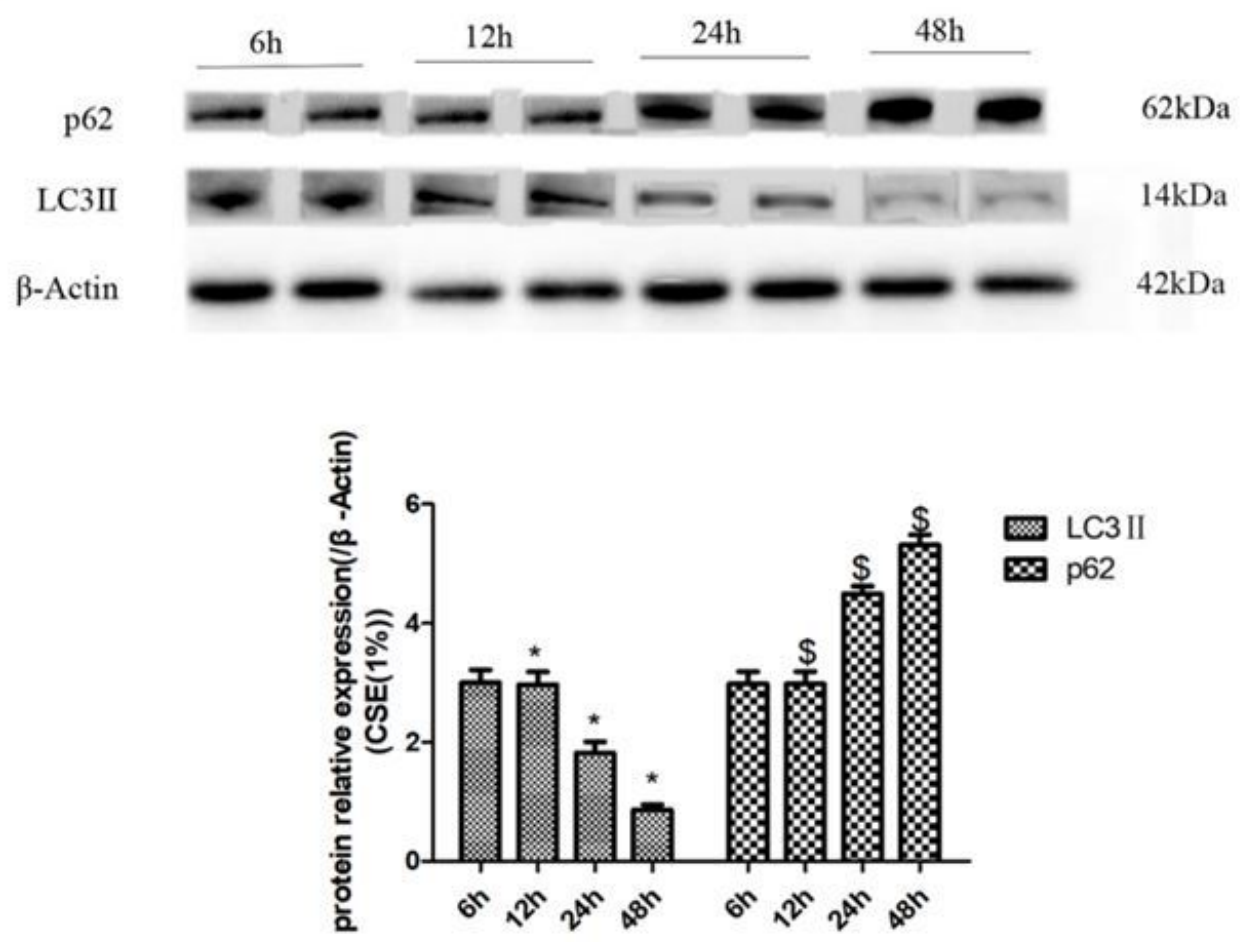

Figure 5

Please see the Manuscript file for the complete figure caption. 
A

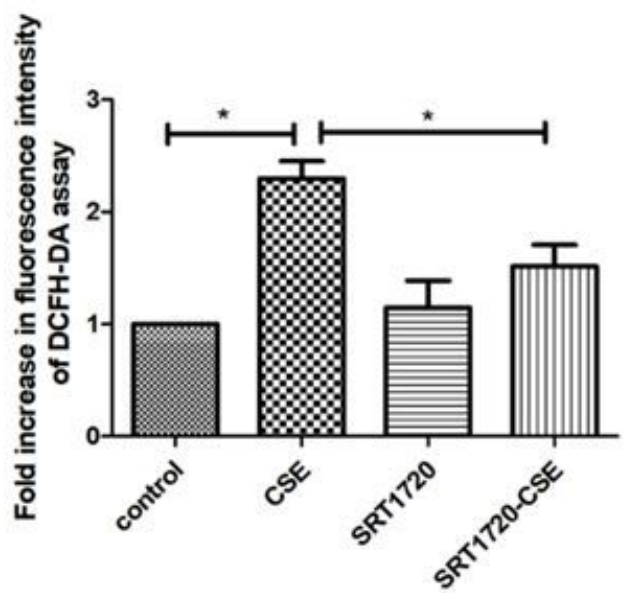

B

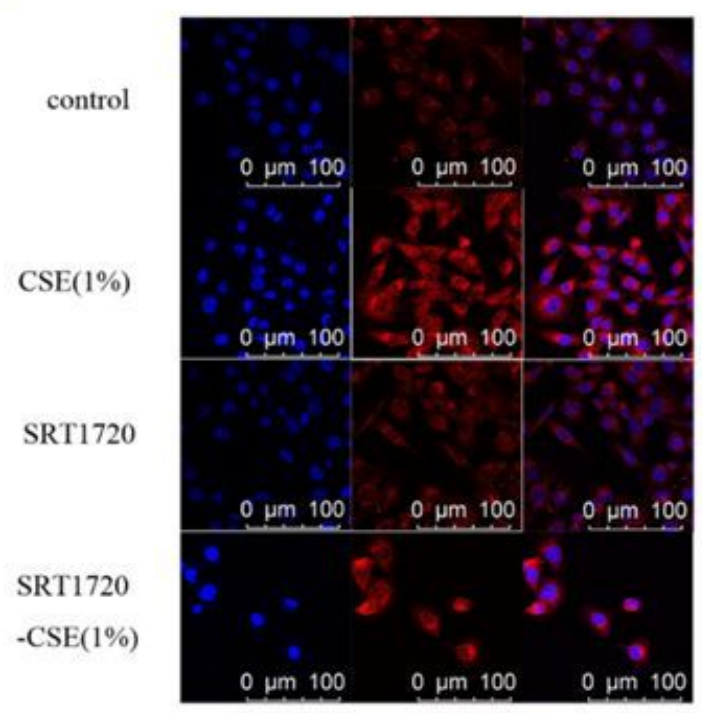

C
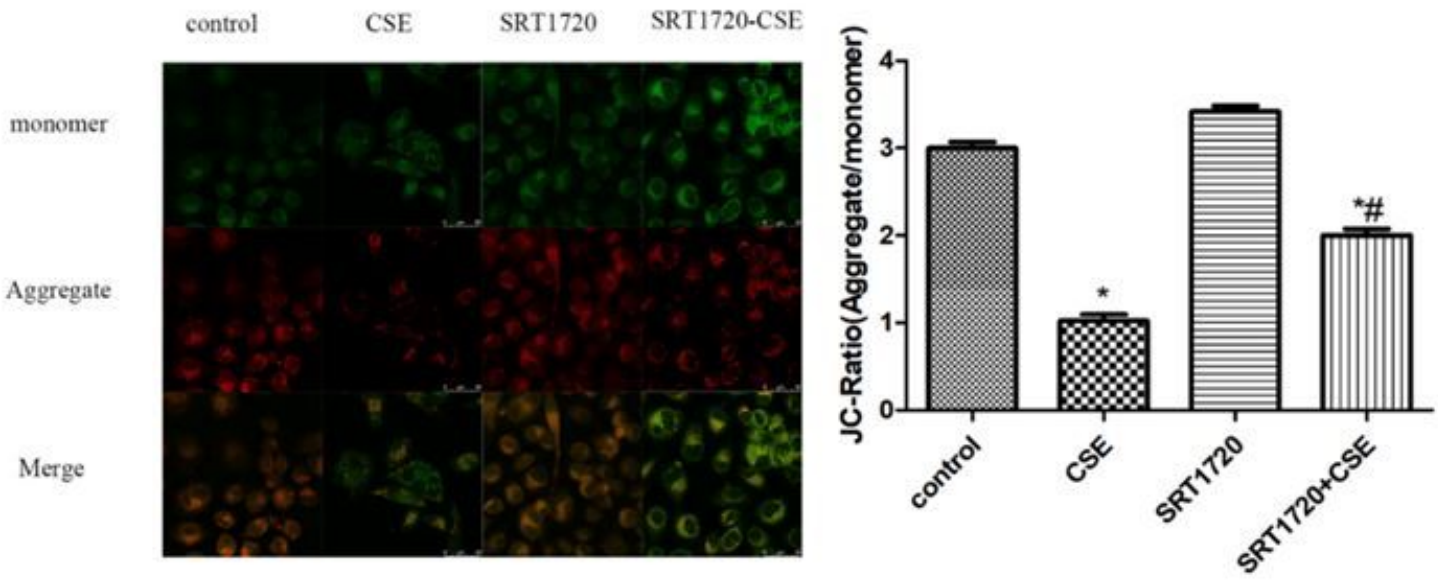

$\mathrm{D}$

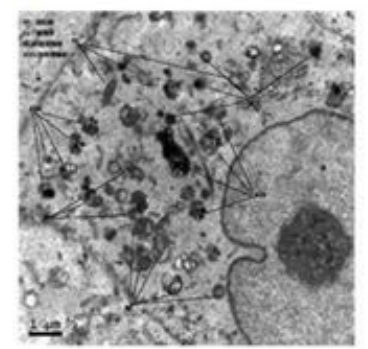

control

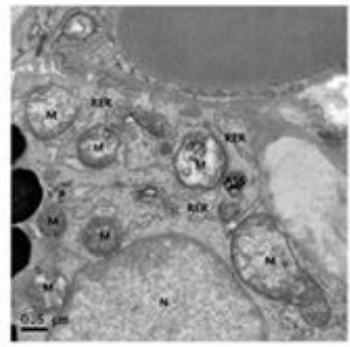

CSE

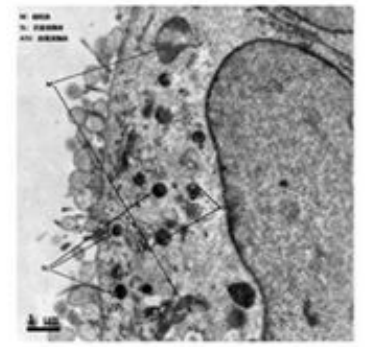

SRT 1720

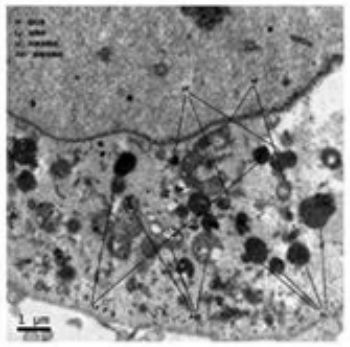

SRT1720-CSE

Figure 6

Please see the Manuscript file for the complete figure caption. 
A

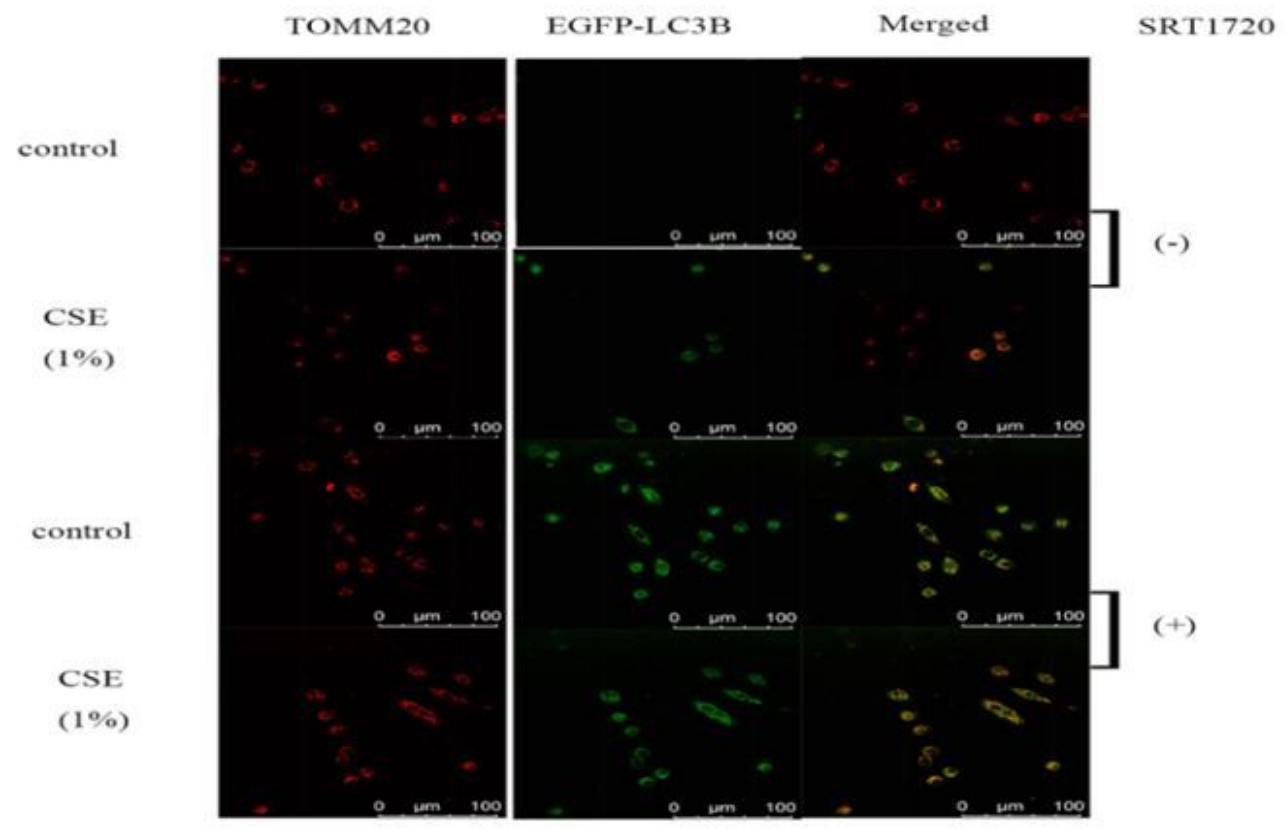

B
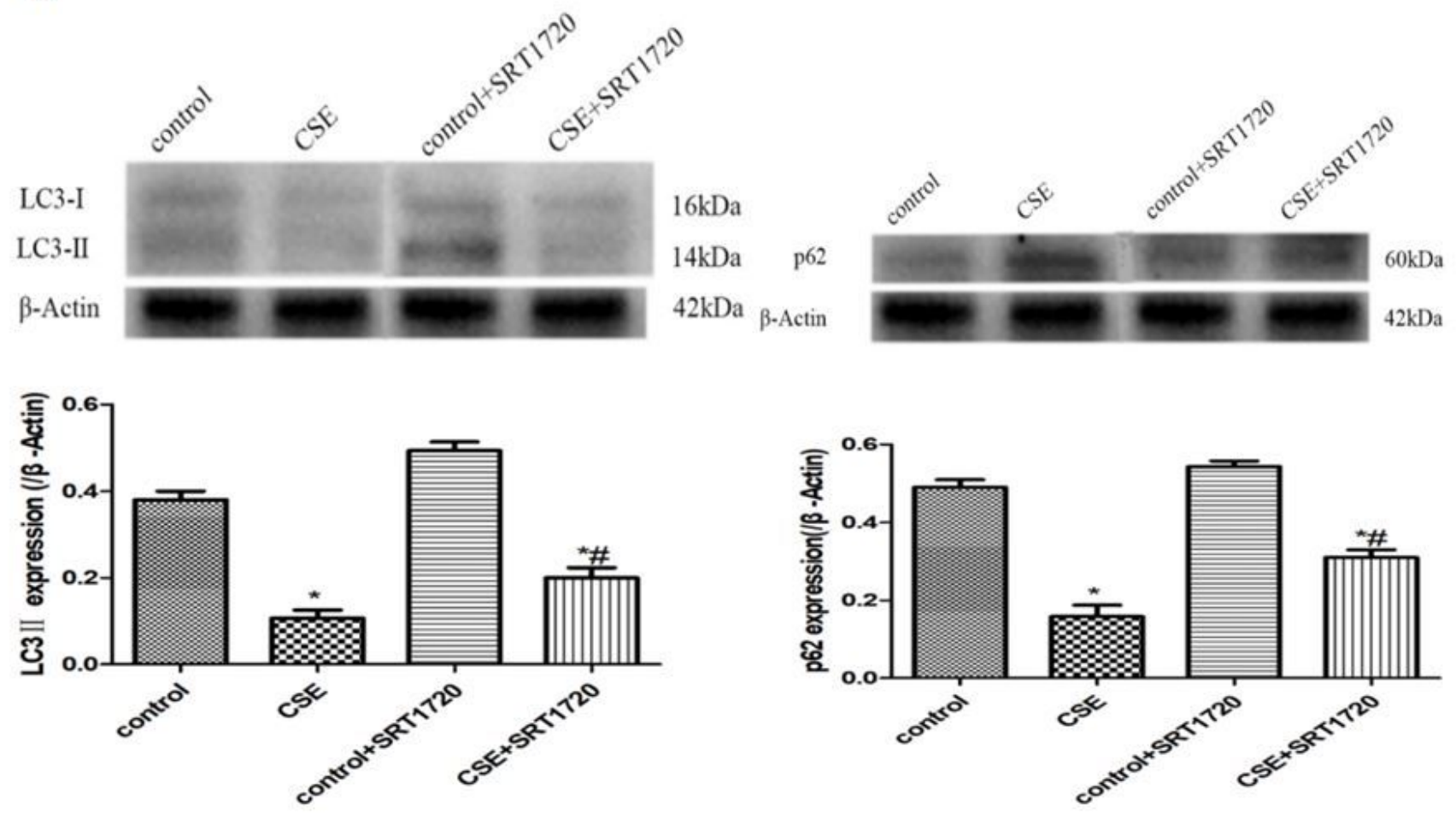

Figure 7

Please see the Manuscript file for the complete figure caption. 
A

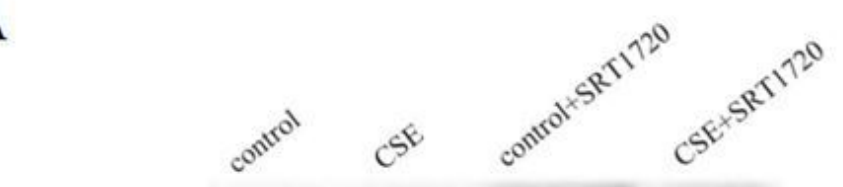

Sirtl

Ac-Foxo3A

Foxo3A

Pink1

$\beta$-Actin

B

$\mathrm{D}$
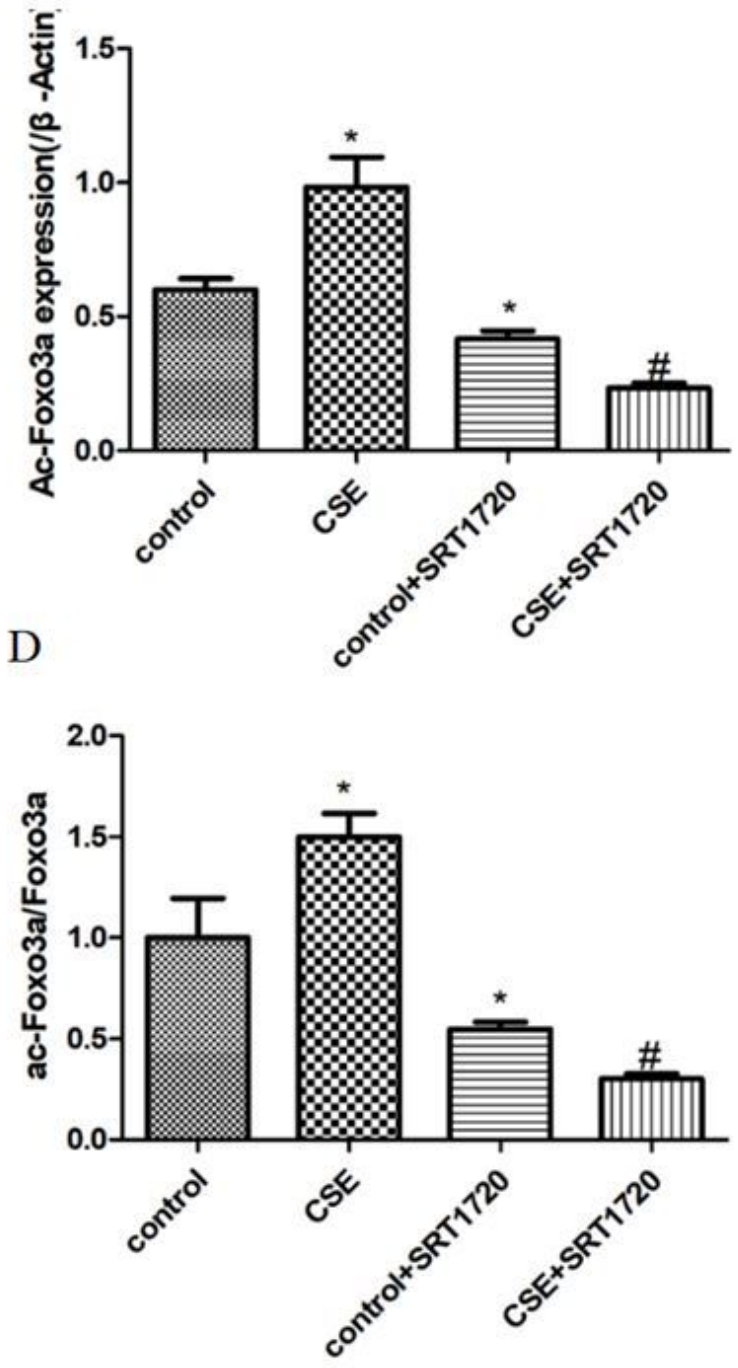

$120 \mathrm{kDa}$

$72 \mathrm{kDa}$

$72 \mathrm{kDa}$

$66 \mathrm{kDa}$

$42 \mathrm{kDa}$

$\mathrm{C}$

E
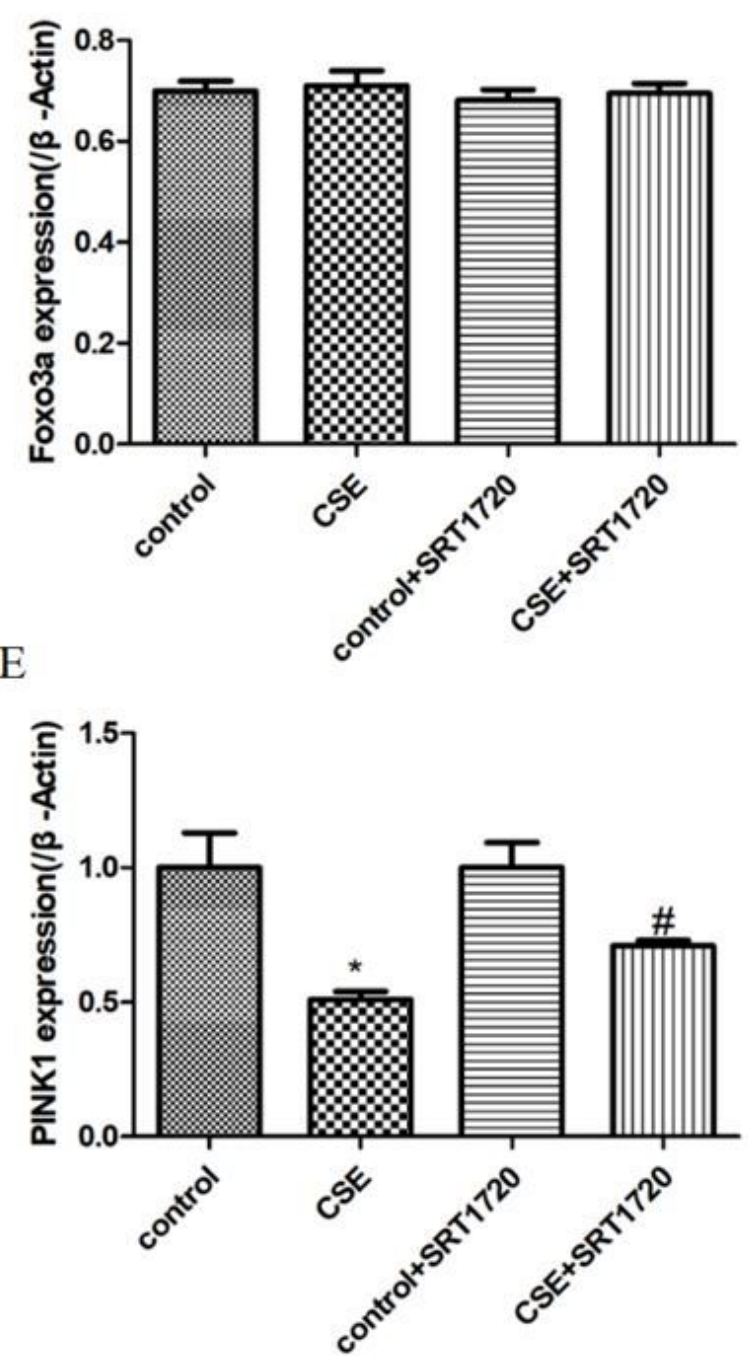

Figure 8

Please see the Manuscript file for the complete figure caption. 